\title{
Development of high-precision La isotope analysis by MC-ICP-MS
}

\author{
MITSURU SUZUKI, TSUYOSHI IIZUKA, KOTA \\ YAMAMOTO AND TAKAFUMI HIRATA
}

The University of Tokyo

Presenting Author: suzuki-mitsuru@eps.s.u-tokyo.ac.jp

The long-lived radionuclide ${ }^{138} \mathrm{La}$ is of exclusive supernova origin and therefore can be used as a tracer of supernova-derived material in the early Solar System. However, the very low isotope abundance of ${ }^{138} \mathrm{La} \quad(0.09 \%)$ makes its precise measurement difficult. Shen and Lee (2003) [1] successfully detected La isotope variation among CAIs using TIMS. Yet, the analytical precision in the pioneering work $(2 \sigma=8-16 \varepsilon)$ is not high enough for investigating potential isotope variations among whole-rock meteorites. In this study, we have developed a method for precise La isotope analysis using MC-ICP-MS. This has been achieved by (1) using $10^{13}$ and $10^{10} \Omega$ amplifiers on 138 and $139 \mathrm{amu},(2)$ correcting for instrumental mass bias by the $\mathrm{Nd}$ external calibration technique, and (3) developing a novel chemical separation scheme for effective removal of isobaric elements, $\mathrm{Ba}$ and $\mathrm{Ce}$.

The precision of $\mathrm{La}$ isotope measurements was tested by analyzing a La standard solution NIST $3127 \mathrm{a}$. The use of $10^{13}$ and $10^{10} \Omega$ amplifiers improved the precision by a factor of two compared with using $10^{11} \Omega$ amplifier. The external calibration technique improved the precision by an order of magnitude compared with the standard bracketing method. As a result of these improvements, an internal precision of $\pm 1.4 \varepsilon$ (2SE) was achieved. We also found that ${ }^{138} \mathrm{Ba}$ and ${ }^{138} \mathrm{Ce}$ isobaric interferences can be accurately corrected when $\mathrm{Ba} / \mathrm{La}$ and $\mathrm{Ce} / \mathrm{La}$ are as low as $2 \times 10^{-3}$ and $2 \times 10^{-4}$, respectively.

Our La chemical separation technique consists of 3-step column chromatographic procedures. In the first step, matrix elements and $\mathrm{Ba}$ were separated from rare earth elements (REEs) by using AG 50W-X8 (100-200 mesh) cation exchange resin with elution using $\mathrm{HCl}$ and $\mathrm{HNO}_{3}$ successively. In the second step, REEs were further purified from $\mathrm{Ba}$ with TODGA resin $(50-100 \mu \mathrm{m})$ by direct loading from the former column. In the last step, La was separated from other REEs using Ln resin (25$50 \mu \mathrm{m}$ ) by eluting with $0.15 \mathrm{M} \mathrm{HCl}$. For $100 \mathrm{mg} \mathrm{JB}-1 \mathrm{~b}$ basaltic reference material, the total recovery of $\mathrm{La}$ was $>97 \%$ while $\mathrm{Ba} / \mathrm{La}, \mathrm{Ce} / \mathrm{La}, \mathrm{Nd} / \mathrm{La}$ were $<3 \times 10^{-4},<5 \times 10^{-5}$ and $<2 \times 10^{-4}$, respectively.

[1] Shen and Lee (2003), ApJL 596, L109-L112. 